\title{
Study of $\mathrm{e}^{+} \mathrm{e}^{-}$annihilation into hadrons with the SND detector at the VEPP-2000 collider
}

\author{
V. P. Druzhinin ${ }^{a b}$, M. N. Achasov ${ }^{a b}$, A. Yu. Barnyakov ${ }^{a b}$, A. .A. Baykov ${ }^{a b}$, \\ K. I. Beloborodov ${ }^{a b}$, A. V. Berdyugin ${ }^{a b}$, A. G. Bogdanchikov ${ }^{a}$, A. A. Botov ${ }^{a}$, \\ T. V. Dimova ${ }^{a b}$, V. B. Golubev ${ }^{a b}$, L. V. Kardapoltsev ${ }^{a b}$, A. G. Kharlamov ${ }^{a b}$, \\ A. A. Korol ${ }^{a b}$, D. P. Kovrizhin ${ }^{a b}$, A. S. Kupich ${ }^{a}$, R. A. Litvinov ${ }^{a}$, K. A. Martin ${ }^{a}$, \\ N. A. Melnikova ${ }^{a b}$ A. E. Obrazovsky ${ }^{a}$, E. V. Pakhtusova ${ }^{a}$, K. V. Pugachev ${ }^{a b}$, \\ S. I. Serednyakov ${ }^{a b}$, D. A. Shtol ${ }^{a b}$, Z. K. Silagadze ${ }^{a b}$, I. K. Surin ${ }^{a b}$, Yu. V. Usov ${ }^{a b}$ and \\ V. N. Zabin ${ }^{a}$. \\ ${ }^{a}$ Budker Institute of Nuclear Physics, Novosibirsk, 630090, Russia \\ ${ }^{b}$ Novosibirsk State University, Novosibirsk, 630090, Russia \\ E-mail: druzhinineinp.nsk.su
}

\begin{abstract}
We present recent results on study of exclusive processes of $e^{+} e^{-}$annihilation into hadrons with the SND detector. Data were collected at the VEPP-2000 $e^{+} e^{-}$collider in the center-of-mass energy range from 0.3 to $2 \mathrm{GeV}$. In particular, we have measured the cross sections for $e^{+} e^{-} \rightarrow$ $\pi^{+} \pi^{-}$and $n \bar{n}$, studied the radiative decays of excited light vector mesons to $\pi^{0} \gamma$ and $\eta \pi^{0} \gamma$, and performed a search for the rare processes of $e^{+} e^{-}$annihilation into $C$-even resonances $\eta$ and $f_{1}(1285)$.
\end{abstract}

European Physical Society Conference on High Energy Physics - EPS-HEP2019 -

10-17 July, 2019

Ghent, Belgium

${ }^{*}$ Speaker. 


\section{Introduction}

SND $[1,2,3,4]$ is the universal nonmagnetic detector consisting of a nine-layer drift chamber, aerogel Cherenkov counters, a three-layer spherical electromagnetic calorimeter with $1640 \mathrm{NaI}(\mathrm{Tl})$ crystals, and a muon system.

SND collected data at two $e^{+} e^{-}$colliders: VEPP-2M [5] during 1996-2000 in the center-ofmass energy range 0.36-1.38 GeV and VEPP-2000 [6] starting from 2010 in the energy range 0.3-2.0 GeV. During the 2010-2013 data taking period the luminosity was limited by the deficit of positrons. From 2014 the VEPP-2000 accelerator complex was under reconstruction. The experiments were restarted by the end of 2016 with a new 10 times more intensive positron source. The data sample with an integrated luminosity of about $70 \mathrm{pb}^{-1}$ has been collected during 2010-2013 and about $200 \mathrm{pb}^{-1}$ in 2017-2019. The achieved luminosity near $2 \mathrm{GeV}$ is about $4 \times 10^{31} \mathrm{~cm}^{-2} \mathrm{~s}^{-1}$.

Main physics task of the SND experiment is the study of all possible processes of $e^{+} e^{-}$annihilation into hadrons below $2 \mathrm{GeV}$. In particular, these measurements are used to obtain the total hadronic cross section needed for Standard Model calculation of the anomalous magnetic moment of muon and running electromagnetic coupling constant. A detailed study of dynamic of exclusive processes is also performed. In this report we present SND results on the processes $e^{+} e^{-} \rightarrow \pi^{+} \pi^{-}$, $e^{+} e^{-} \rightarrow n \bar{n}, e^{+} e^{-} \rightarrow \pi^{0} \gamma, e^{+} e^{-} \rightarrow \eta \pi^{0} \gamma, e^{+} e^{-} \rightarrow \eta$, and $e^{+} e^{-} \rightarrow f_{1}(1285)$.

\section{Measurement of the $\mathrm{e}^{+} \mathbf{e}^{-} \rightarrow \pi^{+} \pi^{-}$cross section}

The process $e^{+} e^{-} \rightarrow \pi^{+} \pi^{-}$is very important for calculation of the hadronic contribution to the $(g-2)$ of muon. There are many measurements of this process, some of them have systematic uncertainty less than $1 \%$.

Our preliminary measurement is based on $4.7 \mathrm{pb}^{-1}$ data, $10 \%$ of the full SND data set in the energy region under study. The event selection is based on excellent $e / \pi$ separation provided by the three layer SND calorimeter. Figure 1 represents the measured $e^{+} e^{-} \rightarrow \pi^{+} \pi^{-}$cross section. The currently achieved systematic uncertainty is $0.8 \%$. The different sources of the uncertainty are listed in Table 1. The curve in Fig. 1 is the result of the fit to data with the vector-meson-dominance
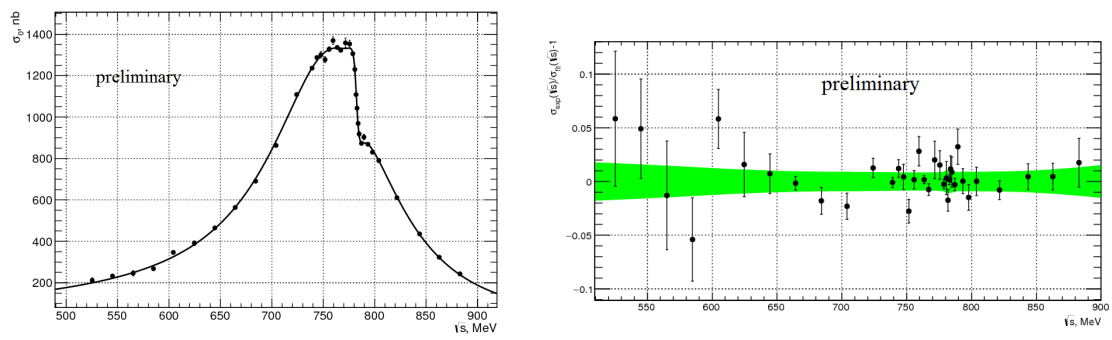

Figure 1: Left panel: The $e^{+} e^{-} \rightarrow \pi^{+} \pi^{-}$cross section measured by SND. The curve is the result of the VMD fit. Right panel: The relative difference between the SND $e^{+} e^{-} \rightarrow \pi^{+} \pi^{-}$data and the fit to the data. The band represents the statistical and systematic uncertainty of the fit combined in quadrature.

(VMD) model including the $\rho(770), \omega(782)$, and $\rho(1450)$ resonances. The model describes data 
Table 1: The sources of systematic uncertainties on the $e^{+} e^{-} \rightarrow \pi^{+} \pi^{-}$cross section (in \%).

\begin{tabular}{lcc}
\hline Source & $<0.6 \mathrm{GeV}$ & $0.6-0.9 \mathrm{GeV}$ \\
\hline Trigger & 0.5 & 0.5 \\
Selection criteria & 0.5 & 0.5 \\
$e / \pi$ separation & 0.6 & 0.6 \\
Nucl. interaction & 0.2 & 0.2 \\
Theory & 0.2 & 0.2 \\
\hline Total & 0.9 & 0.8 \\
\hline
\end{tabular}
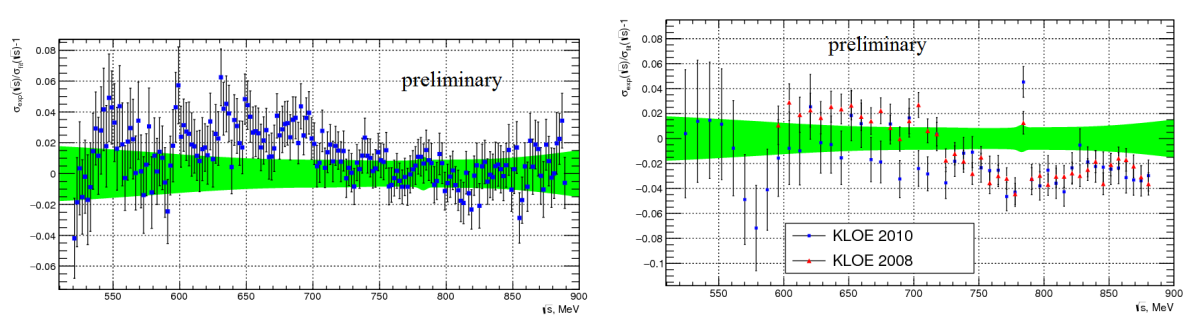

Figure 2: The relative difference between the BABAR [9] (left panel) and KLOE [10, 11] (right panel) $e^{+} e^{-} \rightarrow \pi^{+} \pi^{-}$data and the SND fit. The band represents the statistical and systematic uncertainty of the SND fit combined in quadrature.

well, the obtained resonance parameters are in reasonable agreement with the previous SND measurement [7] and Particle Data Group table [8].

The difference between the currently most accurate BABAR [9] and KLOE [10, 11] $e^{+} e^{-} \rightarrow$ $\pi^{+} \pi^{-}$data and the SND fit is shown in Fig. 2, where the green band represents the statistical and systematic uncertainties of the SND fit combined in quadrature. The systematic difference is observed between the SND and BABAR data below $0.7 \mathrm{GeV}$ and between the SND and KLOE data above $0.7 \mathrm{GeV}$.

We also calculate the contribution to the muon anomalous magnetic moment from the $e^{+} e^{-} \rightarrow$ $\pi^{+} \pi^{-}$channel in the energy region $0.53-0.88 \mathrm{GeV}$ using the new SND data. It is equal to $(411.8 \pm$ $1.0 \pm 3.7) \times 10^{-10}$. This value is in good agreement with the previous SND result [7] (408.9 \pm $1.3 \pm 5.3) \times 10^{-10}$ and the BABAR result [9] $(414.9 \pm 0.3 \pm 2.1) \times 10^{-10}$.

\section{Study of the process $\mathrm{e}^{+} \mathrm{e}^{-} \rightarrow \mathbf{n} \overline{\mathbf{n}}$}

The process $e^{+} e^{-} \rightarrow n \bar{n}$ was previously measured by FENICE [12] and SND [13] using the 2011-2012 data set. The new SND measurement is based on 2017 data and uses a different method of signal-background separation compared with Ref. [13].

The time distribution of the calorimeter trigger signal is analyzed to separate signal from background. This distribution is shown in Fig. 3 for two energy regions. It is fitted by a sum of distributions for signal, cosmic background, and beam plus $e^{+} e^{-}$annihilation background. The latter is estimated from data recorded below the $n \bar{n}$ threshold. The $n \bar{n}$ time distribution is shifted relative to other $e^{+} e^{-}$annihilation events due to small antineutron velocity. 

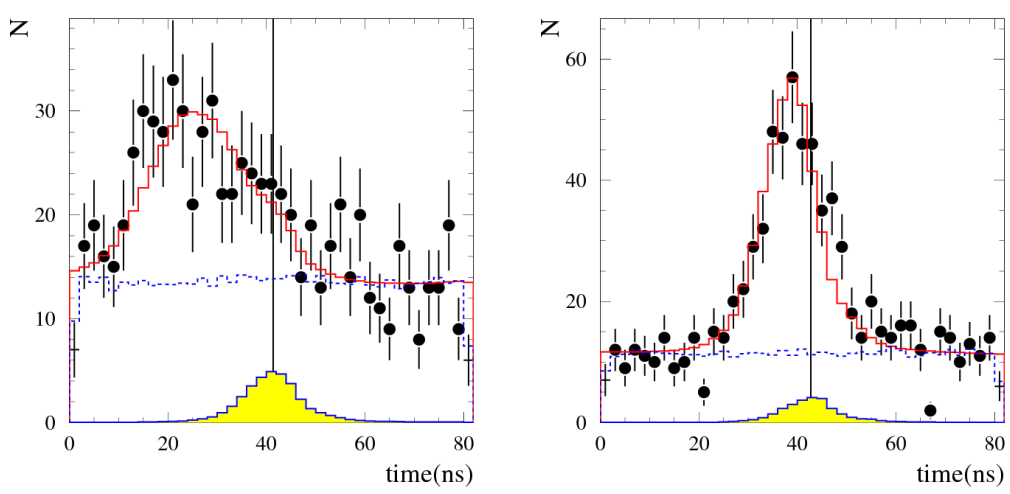

Figure 3: The time distribution of the calorimeter trigger signal for $n \bar{n}$ candidates in data (points with error bars) for the energy regions: $1.880-1.884 \mathrm{GeV}$ (left panel) and 1.90-1.94 GeV (right panel). The solid histogram represents the result of the fit with a sum of signal and background distributions. The dashed histogram shows cosmic background, while the shaded histogram represents $e^{+} e^{-}$annihilation background.
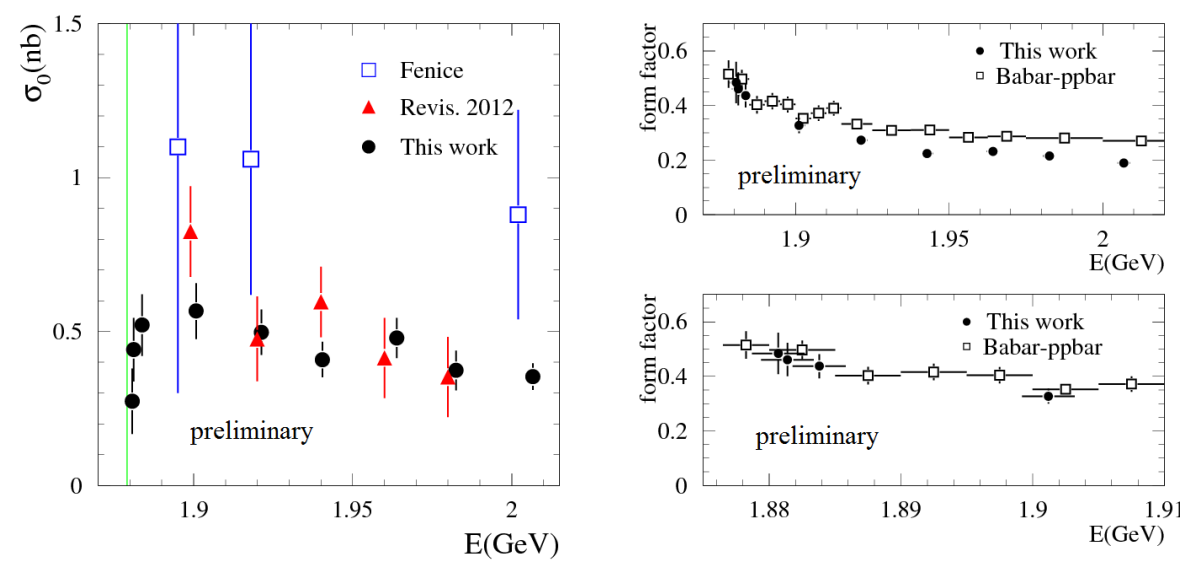

Figure 4: Left panel: The $e^{+} e^{-} \rightarrow n \bar{n}$ cross section measured by SND using the 2012 (triangles) and 2017 (circles) data set in comparison with the FENICE results [12] (squares). Right panel: The neutron effective form factor measured by SND (circles) in comparison with the proton effective form factor measured by BABAR [14] (squares).

The measured cross section is shown in Fig. 4 (left). The statistical accuracy of the measurement is significantly improved compared with the previous SND measurement [13]. However the new SND result is lower than the previous one by about $30 \%$ at $1.9 \mathrm{GeV}$ and by two times near 2 $\mathrm{GeV}$. The main reasons are underestimated beam background and not quite correct MC simulation. The triangles in the plot represent the revised 2012 data, which are in agreement with the new measurement.

The systematic uncertainty on the cross section is estimated to be about $20 \%$, mainly due to MC simulation. Currently we are working to decrease it. 
The $e^{+} e^{-} \rightarrow n \bar{n}$ cross section depends on two form factors, magnetic and electric. From the measured cross section we determine the so-called effective form factor, which is shown in Fig. 4 (right) in comparison with BABAR measurement of the proton form factor. It is seen that the proton and neutron effective form factors are close to each other in the near-threshold region. The neutron form factor becomes lower than the proton one with increase of the energy.
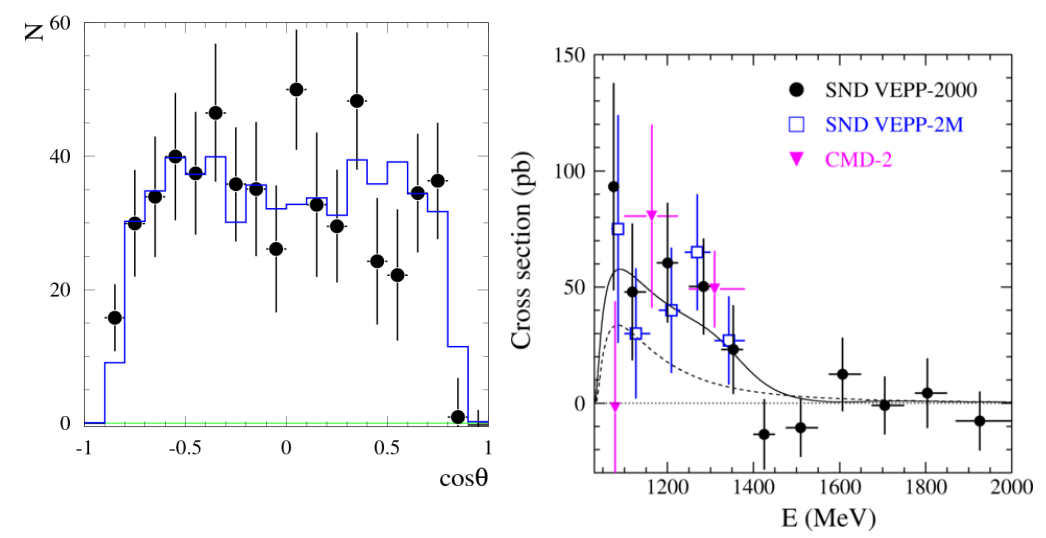

Figure 5: Left panel: The antineutron polar angle distribution (points with error bars). The histogram represent the simulated distribution in the model with $G_{E}=0$. Right panel: The $e^{+} e^{-} \rightarrow \pi^{0} \gamma$ cross section measured by SND at VEPP-2000 [15] in comparison with the previous measurements: SND@VEPP-2M [16], and CMD-2 [17]. The solid curve is the result of the fit in the VMD model, while the dashed curve represents the contribution the $\rho(700), \omega(782)$, and $\phi(1020)$ resonances.

The ratio of the form factors can be determined from the analysis of the antineutron polar angle distribution. The measured distribution shown in Fig. 5 (left) is well described by the model with $G_{E}=0$. The dominance of the $G_{E}$ term in the cross section is excluded. It should be noted that for proton $\left|G_{E} / G_{M}\right|$ is about 1.5 in this energy region.

\section{Radiative processes $\mathbf{e}^{+} \mathbf{e}^{-} \rightarrow \pi^{0} \gamma$ and $\mathbf{e}^{+} \mathbf{e}^{-} \rightarrow \eta \pi^{0} \gamma$}

The process $e^{+} e^{-} \rightarrow \pi^{0} \gamma$ was carefully studied below $1.4 \mathrm{GeV}$ at the VEPP-2M collider by SND [16] and CMD-2 [17]. In these experiments, the evidence of the nonzero contribution of excited vector resonances to the $e^{+} e^{-} \rightarrow \pi^{0} \gamma$ cross section in the energy region 1.075-1.4 GeV was obtained. The new SND analysis [15] is based on data with an integrated luminosity of $41 \mathrm{pb}^{-1}$ recorded in 2010-2012 in the range 1.075-2 GeV.

The measured $e^{+} e^{-} \rightarrow \pi^{0} \gamma$ cross section is shown in Fig. 5 (right) in comparison with the previous SND and CMD-2 measurements. Below $1.4 \mathrm{GeV}$ the obtained cross section is about $50 \mathrm{pb}$ and agrees with the previous SND and CMD 2 results. The curve is the result of the fit to the all SND and CMD-2 data with the VMD model, which includes the contributions of excited vector states. To explain the cross section energy dependence, the contribution of the $\rho(1450)$ and $\omega(1420)$ resonances is required with a significance of $4 \sigma$.

The process $e^{+} e^{-} \rightarrow \eta \pi^{0} \gamma$ above $1.05 \mathrm{GeV}$ is studied for the first time. The five-photon final state is used for the analysis. In this process, there is a significant contribution of the $\omega \eta$ 

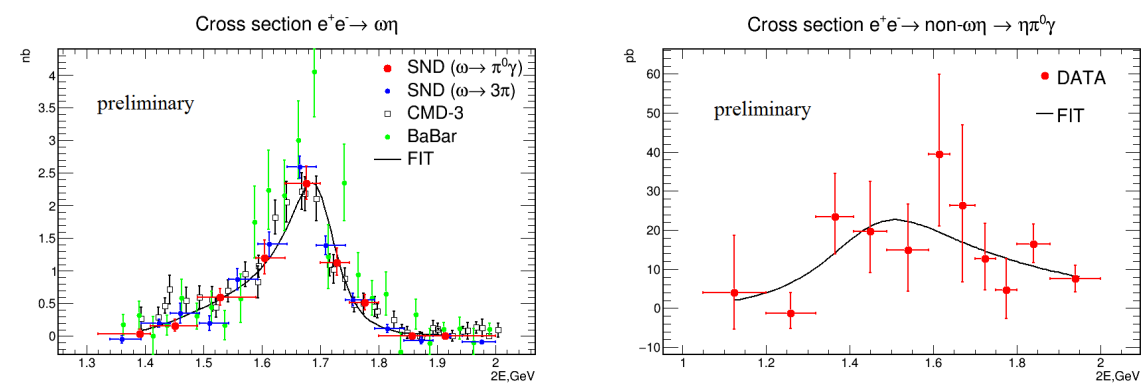

Figure 6: Left panel: The $e^{+} e^{-} \rightarrow \omega \eta$ cross section measured by SND in the $\eta \pi^{0} \gamma$ final state in comparison with the SND [18] and CMD-3 [19] measurements in the $\pi^{+} \pi^{-} \pi^{0} \eta$ final state. Right panel: The non- $\omega \eta$ $e^{+} e^{-} \rightarrow \eta \pi^{0} \gamma$ cross section measured by SND.

intermediate state, which is seen as a peak at $\omega$ mass in the $\pi^{0} \gamma$ mass distribution. The non$\omega \eta$ signal is also observed. It may arise from the processes $e^{+} e^{-} \rightarrow a_{0}(1450) \gamma$ and $a_{2}(1320) \gamma$. Figure 6 represents the measured $e^{+} e^{-} \rightarrow \omega \eta$ cross section in comparison with the SND and CMD3 measurements in the decay mode $\omega \rightarrow 3 \pi$ and the first measurement of the non- $\omega \eta$ cross section in the energy range $1.05-2.00 \mathrm{GeV}$.

\section{Search for direct production of $\mathrm{C}$-even resonances in $\mathrm{e}^{+} \mathrm{e}^{-}$annihilation}

The direct production of $C$-even resonances in $e^{+} e^{-}$annihilation is a rare process suppressed by $\alpha^{2}$ compared with single-photon annihilation. For spin- 0 resonances there is additional helicity suppression.

The search of the process $e^{+} e^{-} \rightarrow \eta$ is performed using a special $650 \mathrm{nb}^{-1}$ data sample recorded in 2018 at the center of mass energy equal to the $\eta$ mass. The visible $\eta$ line shape is determined by the beam energy spread. The beam energy and spread were measured with a special system based on Compton backscattering of laser photons off the electron beam. The decay mode $\eta \rightarrow 3 \pi^{0}$ is used for analysis, in which the single photon annihilation background is absent. Zero signal events have been selected. Since the $e^{+} e^{-} \rightarrow \eta$ is proportional to the $\eta \rightarrow e^{+} e^{-}$branching fraction, the $90 \%$ confidence level upper limit $B\left(\eta \rightarrow e^{+} e^{-}\right)<7 \times 10^{-7}$ [20] is set, which improves the previous limit of the HADES Collaboration [21] by a factor of 3 . The theoretical prediction for this decay is about $5 \times 10^{-9}$ (see [22] and references therein).

The predicted branching fraction for the axial vector resonance $f_{1}$ decay to $e^{+} e^{-}$is $(3.5 \pm$ 1.8) $\times 10^{-9}$ [23]. The corresponding $f_{1}$ production cross section is relatively large, about $30 \mathrm{pb}$.

We analyze the $15 \mathrm{pb}^{-1}$ data sample recorded in the energy range $1.2-1.4 \mathrm{GeV}$. About $4 \mathrm{pb}^{-1}$ were collected at the $f_{1}$ maximum. The decay mode $f_{1} \rightarrow \eta \pi^{0} \pi^{0} \rightarrow 6 \gamma$, which is not produced in single photon annihilation, is used for the analysis.

After applying selection and background suppression cuts, two events are observed at the $f_{1}$ resonance peak and zero events beyond the resonance. The expected background from the processes $e^{+} e^{-} \rightarrow \omega \pi^{0}, \eta \gamma$, and $\omega \pi^{0} \pi^{0}$ does not exhibit the resonance behavior and is about 0.25 events at the $f_{1}$ peak. The observed two events correspond to the cross section of $43_{-23}^{+32} \mathrm{pb}$ and the $f_{1} \rightarrow e^{+} e^{-}$branching fraction $\left(4.9_{-2.6}^{+3.6}\right) \times 10^{-9}$ [24]. The significance of the $f_{1}$ signal is $2.4 \sigma$. 
Acknowledgments. This work is supported in part by the RFBR grant 18-02-00147.

\section{References}

[1] M. N. Achasov et al., Nucl. Instrum. Methods Phys. Res., Sect. A 598, 31 (2009).

[2] V. M. Aulchenko et al., Nucl. Instrum. Methods Phys. Res., Sect. A 598, 102 (2009).

[3] A. Y. Barnyakov et al., JINST 9, C09023 (2014).

[4] V. M. Aulchenko et al., Nucl. Instrum. Methods Phys. Res., Sect. A 598, 340 (2009).

[5] I. A. Koop et al., in Proceedings of the Workshop on Physics and Detectors for DAPHNE, Frascati, Italy, 1999 (Frascati, 1999), p. 393.

[6] A. Romanov et al., in Proceedings of Particle Accelerator Conference PAC 2013, Pasadena, CA USA, 2013, p. 14.

[7] M. N. Achasov et al. (SND Collaboration), J. Exp. Theor. Phys. 103, 380 (2006).

[8] M. Tanabashi et al. (Particle Data Group), Phys. Rev. D 98, 010001 (2018).

[9] J. P. Lees et al. (BaBar Collaboration), Phys. Rev. D 86, 032013 (2012).

[10] F. Ambrosino et al. (KLOE Collaboration), Phys. Lett. B 670, 285 (2009).

[11] F. Ambrosino et al. (KLOE Collaboration), Phys. Lett. B 700, 102 (2011).

[12] A. Antonelli et al. (FENICE Collaboration), Nucl. Phys. B 517, 3 (1998).

[13] M. N. Achasov et al. (SND Collaboration), Phys. Rev. D 90, no. 11, 112007 (2014)

[14] J. P. Lees et al. (BaBar Collaboration), Phys. Rev. D 87, 092005 (2013).

[15] M. N. Achasov et al. (SND Collaboration), Phys. Rev. D 98, 112001 (2018).

[16] M. N. Achasov et al. (SND Collaboration), Phys. Rev. D 93, 092001 (2016).

[17] R. R. Akhmetshin et al. (CMD-2 Collaboration), Phys. Lett. B 605, 26 (2005).

[18] M. N. Achasov et al. (SND Collaboration), Phys. Rev. D 99, 112004 (2019).

[19] R. R. Akhmetshin et al. (CMD-2 Collaboration), Phys. Lett. B 773, 150 (2017).

[20] M. N. Achasov et al. (SND Collaboration), Phys. Rev. D 98, 052007 (2018).

[21] G. Agakishiev et al. (HADES Collaboration), Phys. Lett. B 731, 265 (2014).

[22] P. Sanchez-Puertas, arXiv:1709.04792 [hep-ph].

[23] A. I. Milstein and A. S. Rudenko, arXiv:1909.07938 [hep-ph].

[24] M. N. Achasov et al. (SND Collaboration), arXiv:1906.03838 [hep-ex]. 